\title{
Immunomodulation of RA Patients' PBMC with a Multiepitope Peptide Derived from Citrullinated Autoantigens
}

\author{
Smadar Gertel, ${ }^{1,2}$ Gidi Karmon, ${ }^{1,2}$ Sivan Vainer, ${ }^{1,2}$ Ora Shovman, ${ }^{1,2,3}$ Martin Cornillet, ${ }^{4,5}$ \\ Guy Serre, ${ }^{4,5}$ Yehuda Shoenfeld, ${ }^{1,6}$ and Howard Amital ${ }^{1,2,3}$ \\ ${ }^{1}$ Zabludowicz Center for Autoimmune Diseases, Sheba Medical Center, Tel-Hashomer, 5262100 Ramat-Gan, Israel \\ ${ }^{2}$ Sackler Faculty of Medicine, Tel-Aviv University, 6997801 Tel-Aviv, Israel \\ ${ }^{3}$ Department of Internal Medicine B, Sheba Medical Center, 5262100 Tel Hashomer, Israel \\ ${ }^{4}$ Unité Différenciation Epithéliale et Autoimmunité Rhumatoïde, Université de Toulouse-INSERM, Toulouse, France \\ ${ }^{5}$ Laboratoire de Biologie Cellulaire et Cytologie, CHU de Toulouse, Toulouse, France \\ ${ }^{6}$ Incumbent of the Laura Schwarz-Kipp Chair for Research of Autoimmune Diseases, Tel Aviv University, 6997801 Tel Aviv, Israel \\ Correspondence should be addressed to Smadar Gertel; smadar.gertel@sheba.health.gov.il
}

Received 23 January 2017; Revised 19 April 2017; Accepted 11 May 2017; Published 21 June 2017

Academic Editor: Tânia Silvia Fröde

Copyright (C) 2017 Smadar Gertel et al. This is an open access article distributed under the Creative Commons Attribution License, which permits unrestricted use, distribution, and reproduction in any medium, provided the original work is properly cited.

\begin{abstract}
Citrullinated peptides are used for measuring anticitrullinated protein antibodies (ACPA) in rheumatoid arthritis (RA). Accumulation of citrullinated proteins in the inflamed synovium suggests that they may be good targets for inducing peripheral tolerance. In view of the multiplicity of citrullinated autoantigens described as ACPA targets, we generated a multiepitope citrullinated peptide (Cit-ME) from the sequences of major citrullinated autoantigens: filaggrin, $\beta$-fibrinogen, vimentin, and collagen type II. We assessed the ability of Cit-ME or the citrullinated $\beta 60-74$ fibrinogen peptide ( $\beta 60-74$-Fib-Cit) which bears immunodominant citrullinated epitopes (i) to modify cytokine gene expression and (ii) to modulate Treg and Th17 subsets in PBMC derived from newly diagnosed untreated RA patients. RA patient's PBMC incubated with Cit-ME or $\beta 60-74-\mathrm{Fib}-\mathrm{Cit}$, showed upregulation of TGF- $\beta$ expression ( $16 \%$ and $8 \%$, resp.), and increased CD $4^{+}$Foxp $3^{+}$Treg ( $22 \%$ and $19 \%$, resp.). Both peptides were shown to downregulate the TNF- $\alpha$ and IL- $1 \beta$ expression; in addition, Cit-ME reduced CD $3^{+} \mathrm{IL} 17^{+} \mathrm{T}$ cells. We showed that citrullinated peptides can modulate the expression of anti- and proinflammatory cytokines in PBMC from RA patients as well as the proportions of Treg and Th17 cells. These results indicate that citrullinated peptides could be active in vivo and therefore might be used as immunoregulatory agents in RA patients.
\end{abstract}

\section{Introduction}

Rheumatoid arthritis (RA) is an autoimmune disease estimated to affect nearly $1 \%$ of the adult population worldwide. It is characterized by progressive synovial inflammation resulting in irreversible joint destruction [1]. Many of the RA-specific autoantibodies are generated against citrullinated antigens and termed anticitrullinated protein antibodies (ACPA). These autoantibodies present a high diagnostic and prognostic value [2-4]. The targets for ACPA are citrullinated peptides, that is, peptides that underwent posttranslation conversion of arginine to citrulline residues catalyzed by peptidylarginine deiminases (PAD). Currently, citrullinated peptides serve merely as immunosorbents in the ACPA diagnostic assays. The four citrullinated proteins, filaggrin, fibrinogen, vimentin, and collagen type II, have been described as the major autoantigens in RA [5-7].

The pathogenesis of RA appears to be based on inflammatory responses mediated by self-reactive $\mathrm{T}$ and $\mathrm{B}$ cells reacting against citrullinated proteins. In autoimmune conditions, $\mathrm{T}$ cells reactive to self-antigens escape elimination in the thymus and are activated in the periphery where they can damage specific organs.

PAD isoforms are expressed in thymic epithelial cells; however, it is not clear whether a negative selection of citrulline-specific $\mathrm{T}$ cells occurs and whether those $\mathrm{T}$ cell clones manage to escape into the periphery [8]. In addition, the presence of ACPA is associated with RA susceptibility 
alleles such as HLA-DRB1, HLA-DRB4, and HLA-DRB10 genes $[9,10]$. These alleles encode for a specific motif in the peptide-binding pocket of the human leucocyte antigen (HLA) bearing the so-called shared epitope (SE) [11]. Citrullination may enhance the immunogenicity of "altered-self" peptides through increased binding affinity to SE-containing HLA-DRB presented on MHC class II molecules. This may result in loss of tolerance to the related citrullinated antigens, mediated by activation of citrullinespecific-autoreactive $\mathrm{T}$ and $\mathrm{B}$ cells, leading to production of ACPA, then formation of immune complexes in the synovial tissue, and induction of the proinflammatory cytokine secretion by macrophages, all leading to synovial inflammation [9, 12-14].

The inflamed joints in RA contain a large number of $\mathrm{T}$ cells, the majority of which are $\mathrm{CD} 4^{+} \mathrm{T}$ cells [15]. The autoantigen that causes stimulation and expansion of specific $\mathrm{T}$ cell subsets should be present in the joint and presented by the HLA-DR alleles associated with RA. The fact that inflamed joints of RA patients contain large amounts of citrullinated proteins implies that they may be possible targets for tolerance induction [16]. Restoring self-tolerance without causing immunosuppression remains a major challenge.

In view of the multiplicity of citrullinated proteins described as target autoantigens for ACPA, we generated a multiepitope citrullinated peptide (Cit-ME) derived from the sequence of the major prevalent autoantigenic citrullinated proteins, namely, filaggrin, $\beta$-fibrinogen, vimentin, and collagen type II. Previously, we reported that the Cit-ME peptide ameliorates adjuvant-induced arthritis (AIA) in rats via induction of regulatory $\mathrm{T}$ cells and downregulation of Th17 cells $[17,18]$.

The current research analyzed the capacity of the same citrullinated multiepitope peptide, Cit-ME and of an additional single citrullinated fibrinogen peptide bearing immunodominant epitopes, to modify cytokine expression and modulate $\mathrm{T}$ cell subsets in PBMC from patients with RA.

\section{Materials and Methods}

2.1. Human Subjects and Sample Procurement. A total of 30 patients with RA (17 females and 13 males) were included in the study. All patients fulfilled the 2010 American College of Rheumatology (ACR) criteria for RA. The study protocol was approved by the Medical Ethics Review Board of Sheba Medical Center. All participants signed an informed consent form prior to the initiation of the study. We enrolled only newly diagnosed patients. Patients' age was $52.9 \pm 13.7$ (mean \pm standard deviation) years, and $90 \%$ were ACPA positive (Table 1). Blood samples were collected before starting any therapy. The study was designed to test immunomodulation induced by citrullinated peptides and to assess their possible contribution to the pathogenesis of arthritis. The suggested controls were approved biological disease modifying antirheumatic drugs; therefore, the assays were conducted only on PBMC derived from patients with RA.
TABLE 1: Characterization of rheumatoid arthritis patients analyzed in the in vitro assays.

\begin{tabular}{|c|c|c|c|c|}
\hline Patient number & Gender & Age & ACPA status & ACPA value \\
\hline 1 & Female & 35 & Negative & 12 \\
\hline 2 & Female & 58 & Moderate & 49 \\
\hline 3 & Female & 36 & Moderate & 48 \\
\hline 4 & Female & 32 & Weakly positive & 26 \\
\hline 5 & Male & 50 & Highly positive & 138 \\
\hline 6 & Female & 38 & Positive & 78 \\
\hline 7 & Female & 51 & Negative & 4 \\
\hline 8 & Male & 49 & Highly positive & 242 \\
\hline 9 & Female & 50 & Positive & 55 \\
\hline 10 & Female & 50 & Highly positive & 258 \\
\hline 11 & Male & 35 & Highly positive & 195 \\
\hline 12 & Female & 68 & Positive & 61 \\
\hline 13 & Female & 33 & Highly positive & 128 \\
\hline 14 & Male & 53 & Highly positive & 211 \\
\hline 15 & Female & 64 & Highly positive & 600 \\
\hline 16 & Male & 60 & Negative & 3 \\
\hline 17 & Female & 45 & Positive & 41 \\
\hline 18 & Male & 70 & Highly positive & 210 \\
\hline 19 & Male & 67 & Highly positive & 3700 \\
\hline 20 & Male & 57 & Highly positive & 191 \\
\hline 21 & Female & 55 & Highly positive & 171 \\
\hline 22 & Female & 48 & Moderate & 57 \\
\hline 23 & Male & 22 & Highly positive & 121 \\
\hline 24 & Female & 65 & Highly positive & 227 \\
\hline 25 & Male & 58 & Negative & 6 \\
\hline 26 & Female & 75 & Highly positive & 210 \\
\hline 27 & Male & 72 & Highly positive & 210 \\
\hline 28 & Male & 63 & Highly positive & 210 \\
\hline 29 & Male & 60 & Highly positive & 210 \\
\hline 30 & Female & 70 & Highly positive & 150 \\
\hline
\end{tabular}

2.2. Detection of Antictrullinated Protein Antibodies (ACPA). Sera from patients were tested for ACPA using the commercial ELISA QUANTA Lite ${ }^{\circledR}$ CCP3 IgG kit (Inova, USA), according to the manufacturer's instructions. Sera with results $<25 \mathrm{U} / \mathrm{mL}$ are defined as negative, and sera with results $\geq 25 \mathrm{U} / \mathrm{mL}$, as positive.

2.3. Synthetic Peptides and Biotherapeutic Agents. The multiepitope citrullinated peptide Cit-ME (Figure 1) and its noncitrullinated counterpart containing arginine instead of citrulline residues, Non-Cit-ME, were obtained from GL Biochem (Shanghai, China). The citrullinated peptide $\beta$-Fib-Cit

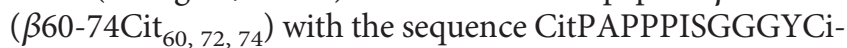
tACit derived from the $\beta$ chain of human fibrinogen [19] and its noncitrullinated counterpart ( $\beta$-Fib-NC) were obtained from NeoMPS (Strasbourg, France). The sequences of $\beta$ Fib-Cit and $\beta$-Fib-NC are also present in part in Cit-ME and Non-Cit-ME, respectively.

All peptides were added to cell culture medium at a concentration of $1.25 \mu \mathrm{g} / \mathrm{mL}$. This concentration was selected 


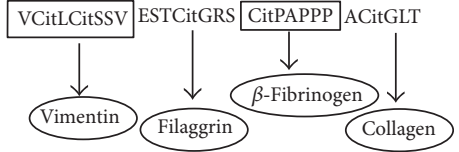

Figure 1: Scheme of Cit-ME peptide. Representative scheme of the multiepitope citrullinated peptide sequence delineated from the major autoantigens of anticitrullinated protein antibody (ACPA). The citrullinated residues are indicated (Cit).

based on previous publications dealing with the capability of autoantigen peptides to induce proliferation at various concentrations of $2.5-10 \mathrm{ug} / \mathrm{mL}[20,21]$. We determined no proliferation at a concentration of $1.25 \mu \mathrm{g} / \mathrm{mL}$ with different citrullinated peptides (data not shown); hence, this concentration was selected to confirm tolerizing regime. In the same way, infliximab or tocilizumab was added to cell culture medium at a concentration of $10 \mu \mathrm{g} / \mathrm{mL}$.

2.4. In Vitro Experiments. Peripheral blood mononuclear cells (PBMC) were isolated from heparinized venous blood using lymphoprep (Amersham). Cells were cultured in RPMI 1640 medium containing 10\% bovine fetal serum supplemented with penicillin $(100 \mathrm{U} / \mathrm{mL})$, streptomycin $(100 \mathrm{~g} / \mathrm{mL}), 2 \mathrm{mmol} / \mathrm{L}$ L-glutamine, and $50 \mu \mathrm{M} 2-\beta$-mercaptoethanol. Cells were incubated for $24-72$ hours at $37^{\circ} \mathrm{C}$ with the experimental peptides, drugs, or medium alone as control.

2.5. Tregs and Th17 Staining. Tregs were identified by the analysis of CD4 and Foxp3 expression. Cells were labeled with anti-CD4 (eBioscience Inc., San Diego, CA, USA), then fixed and permeabilized with Foxp3 detection kit buffers, and stained with anti-Foxp3. In some experiments, anti-CD25 was used to identify $\mathrm{CD} 4^{+} \mathrm{CD} 25^{+} \mathrm{Foxp}^{+}$cells. Th17 cells were determined by addition of phorbol 12-myristate 13acetate (PMA) $(5 \mathrm{ng} / \mathrm{mL})$ and ionomycin $(1 \mu \mathrm{M})$ (SigmaAldrich, Seelze, Germany) to the culture media for 6 hours. Monensin (eBioscience Inc.) was added in the last two hours of stimulation. For Th17 analysis, cells were stained with anti-CD3, followed by fixation and permeabilization (eBioscience Inc.), and then stained intracellularly with anti-IL-17. Cells were analyzed by a flow cytometer (FACScalibur, Becton Dickinson, Franklin Lakes, NJ, USA). Data analysis was conducted using FlowJo software (Tree Star, Ashland, OR, USA).

2.6. Real-Time PCR. RNA was isolated using the Total RNA Purification Plus Kit (Norgen Biotek, ON, CA) according to the manufacturer's instructions. For cDNA synthesis, $1 \mu \mathrm{g}$ total RNA was transcribed with cDNA transcription reagents using the High Capacity cDNA Reverse Transcription Kit (Invitrogen $^{\mathrm{TM}}$, Carlsbad, CA, USA) according to the manufacturer's instructions. Gene expression was measured in real-time PCR performed on a StepOnePlus ${ }^{\mathrm{TM}}$ Real-Time PCR System (Applied Biosystems, Foster City, CA, USA) according to the manufacturer's instructions. Primer sequences (forward and reverse, resp.) were the following: human TGF- $\beta$ 5'-GACACCAACTATTGCTTCAG- $3^{\prime}$ and
5'- CAGGCTCCAAATGTAGGG-3', TNF- $\alpha \quad 5^{\prime}$-CCCAGG GACCTCTCTCTAATCA- $3^{\prime}$ and $5^{\prime}$-GGTTTGCTACAACA TGGGCTACA- ${ }^{\prime}$, IL-1 $\beta$ 5'-TGATGGCTTATTACAGTGG CAATG- $3^{\prime}$ and $5^{\prime}$-GTAGTGGTGGTGGGAGATTCG- $3^{\prime}$, IL $-65^{\prime}$-CAATCTGGATTCAATGAGGAGAC-3' and $5^{\prime}$-TGT TCCTCACTACTCAAATCT-3', IL-8 5'-TGGCAGCCTTC

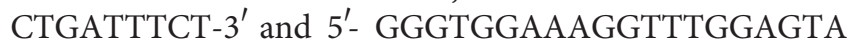
TG- $3^{\prime}$ and CCL- $35^{\prime}$-CCGGTGTCATCTTCCTAACC- ${ }^{\prime}$ and $5^{\prime}$-TTCTGGACCCACTCCTCACT-3', and GAPDH $5^{\prime}$-GA AGGTGAAGGTCGGAGTC- ${ }^{\prime}$ and $5^{\prime}$ - GAAGATGGTGAT GGGATTTC-3'. The expression level of a gene in a given sample was represented as $2^{-\Delta \Delta \mathrm{Ct}}$ where $\Delta \Delta \mathrm{CT}=$ $\left[\Delta \mathrm{CT}_{\text {(experimental) }}\right]-\left[\Delta \mathrm{CT}_{\text {(medium) }}\right]$ and $\Delta \mathrm{CT}=\left[\mathrm{CT}_{\text {(experimental })}\right]$ $-\left[\mathrm{CT}_{\text {(housekeeping) }}\right]$. The GAPDH levels were used to normalize gene expression levels.

2.7. Statistical Analysis. Statistical comparisons were conducted using the two-tailed Mann Whitney $U$ test and Student $t$-test (GraphPad/Prism version 5 software, San Diego, CA, USA).

\section{Results}

3.1. Low Doses of Citrullinated Peptides Change Gene Expression of Anti- and Proinflammatory Cytokines in Cultured PBMC from RA Patients. To detect whether the multiepitope citrullinated peptide Cit-ME and the individual citrullinated peptide $\beta$-Fib-Cit could induce tolerance through immunomodulation of immune cells in RA, we studied their in vitro effects on cytokine gene expression and $\mathrm{T}$ cell subsets of cultured PBMC obtained from RA patients.

The PBMC were cultured for 24 hours in the absence or presence of the citrullinated peptides and as controls their arginine counterparts, at a concentration of $1.25 \mu \mathrm{g} / \mathrm{mL}$ or with infliximab at $10 \mu \mathrm{g} / \mathrm{mL}$. Thereafter, mRNA was isolated from the cells, and the level of TGF- $\beta$, TNF- $\alpha$, and IL- $1 \beta$ gene expression was determined by real-time RT-PCR.

As shown in Figure 2(a), Cit-ME peptide significantly upregulated the TGF- $\beta$ gene expression level in the PBMC $(p=0.04)$ compared to incubation of cells with medium alone (normalization was performed to GAPDH and medium control was considered as 1 ). The peptide $\beta$ Fib-Cit seemed to also upregulate TGF- $\beta$ gene expression, but this was not significant with the small number of patients studied. None of the noncitrullinated peptides produced any upregulation of the TGF- $\beta$ gene expression compared to the medium. In contrast, incubation of cells with infliximab resulted in decreased TGF- $\beta$ expression, compared to medium control.

Concomitantly, the Cit-ME peptide significantly downregulated the proinflammatory TNF- $\alpha$ and IL- $1 \beta$ expression levels as compared to the medium control $(p=0.03$ and $p=0.05$, resp.), as shown in Figure 2(b). The $\beta$-Fib-Cit peptide showed a tendency towards reducing TNF- $\alpha$ and IL- $1 \beta$ levels compared to the medium, but this remained nonsignificant. The noncitrullinated peptides were less potent in reducing TNF- $\alpha$ and IL- $1 \beta$ levels compared to their counterpart citrullinated peptides. Infliximab had no effect on the 


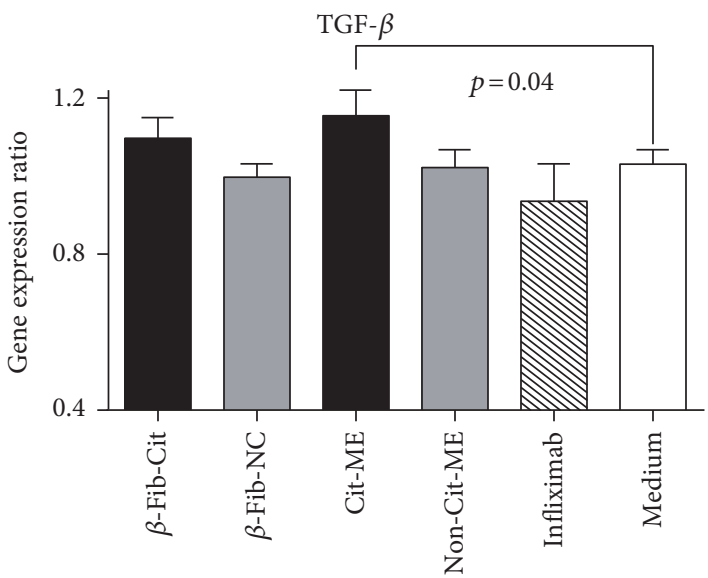

(a)
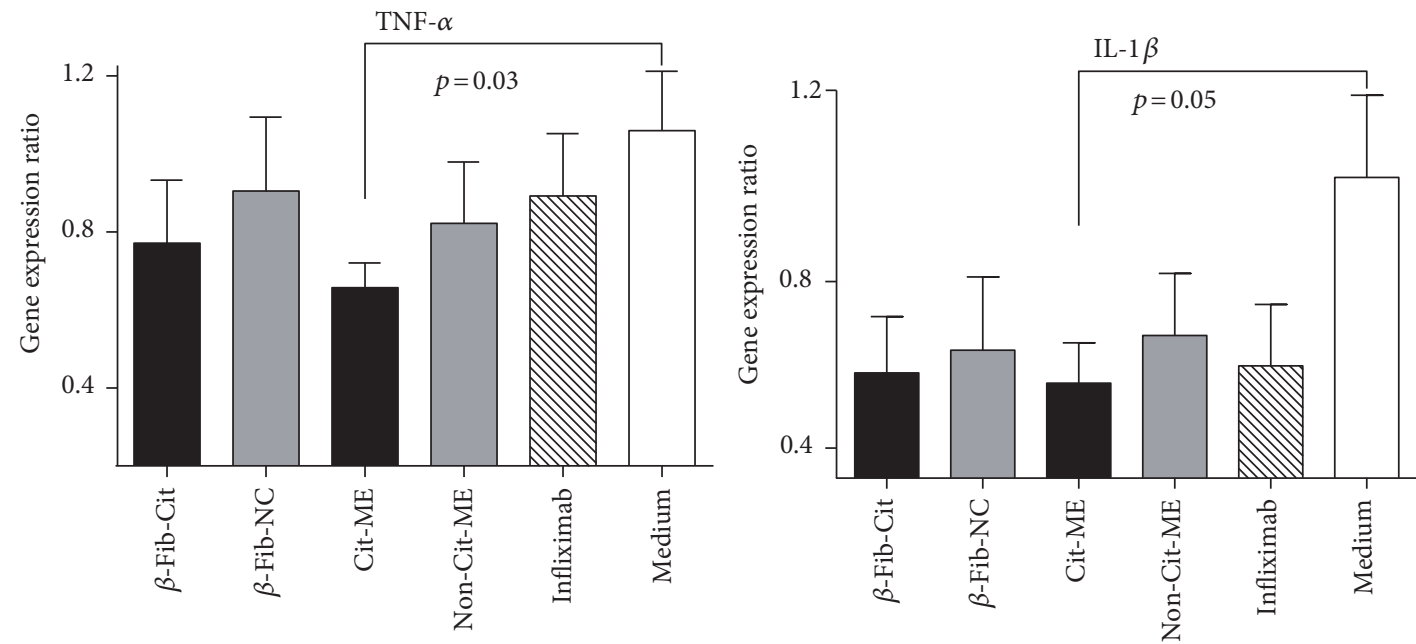

(b)

FIgURE 2: Cit-ME peptide upregulates TGF- $\beta$ and downregulates TNF- $\alpha$ and IL- $1 \beta$ mRNA expression. PBMC of RA patients $(n=12)$ were cultured $\left(5 \times 10^{5}\right.$ cells/well $)$ for 24 hours in the presence of citrullinated peptides $(1.25 \mu \mathrm{g} / \mathrm{mL})$. TGF- $\beta$, TNF- $\alpha$, and IL- $1 \beta$ mRNA gene expression was determined by real-time RT-PCR. Results are presented as the mean \pm SE percentage of gene expression.

TNF- $\alpha$ level but reduced the mean IL-1 $\beta$ level compared to the medium control $(p=0.07)$.

3.2. The Cit-ME Peptide Downregulates IL-6, IL-8, and CCL3 $m R N A$ Expression in Cultured PBMC from RA Patients. Since Cit-ME peptide immunomodulates the expression of essential inflammatory genes, it was of interest to find out whether it might alter the expression of additional related genes. We measured cytokine gene expression typically involved in innate immune responses, namely, IL-6, IL-8, and CCL-3. Figure 3, represents cytokine gene expression levels (normalization was performed to GAPDH and medium control was considered as 1). In accordance with the expression pattern of the proinflammatory TNF- $\alpha$ and IL- $1 \beta$, incubation of Cit-ME with PBMC of RA patients resulted in a significant downregulation in IL-6, IL-8, and CCL-3 gene expression as compared to incubation with medium alone $(p<0.02)$. However, the control peptide Non-Cit-ME could also downregulate significantly the IL-6 expression $(p<0.02)$ as compared to medium control. Whereas, the control peptide NonCit-ME or infiliximab could not downregulate significantly the IL-8 and CCL-3 expression as compared to medium control in cultured RA patients' PBMC.

3.3. The Cit-ME Peptide Affects Treg and Th17 Proportions in Cultured PBMC from RA Patients. The effects of Cit-ME peptide on Treg were analyzed in cultured PBMC from RA patients. PBMC $\left(2 \times 10^{6}\right)$ were cultured with Cit-ME, $\beta$-FibCit, matched noncitrullinated peptides (Non-Cit-ME and $\beta$-Fib-NC), or medium alone. We also tested whether infliximab and tocilizumab could affect Treg proportion as well. Samples were analyzed by flow cytometry 72 hours after incubation, to measure the frequency of $\mathrm{CD} 4^{+} \mathrm{Foxp} 3^{+} \mathrm{T}$ cells. Figure 4(a) shows representative plot of $\mathrm{ACPA}^{+}-\mathrm{RA}$ patient PBMC stained with anti-CD4 and anti-Foxp3. As shown in Figure 4(b), Cit-ME significantly increased the fold change of $\% \mathrm{CD}^{+} \mathrm{Foxp}^{+} \mathrm{T}$ cells $(n=13$, average of $1.22 \pm 0.06)$ compared to the Non-Cit-ME $(n=13$, average of $1.0 \pm 0.07)$ or medium ( $n=12$, considered as 1$)(p=0.02)$, respectively. In addition, incubation with the $\beta$-Fib-Cit peptide resulted in significant increase in fold change of $\% \mathrm{CD} 4^{+} \mathrm{Foxp}^{+}$ $\mathrm{T}$ cells $(n=6$, average of $1.19 \pm 0.07)$ as compared to 

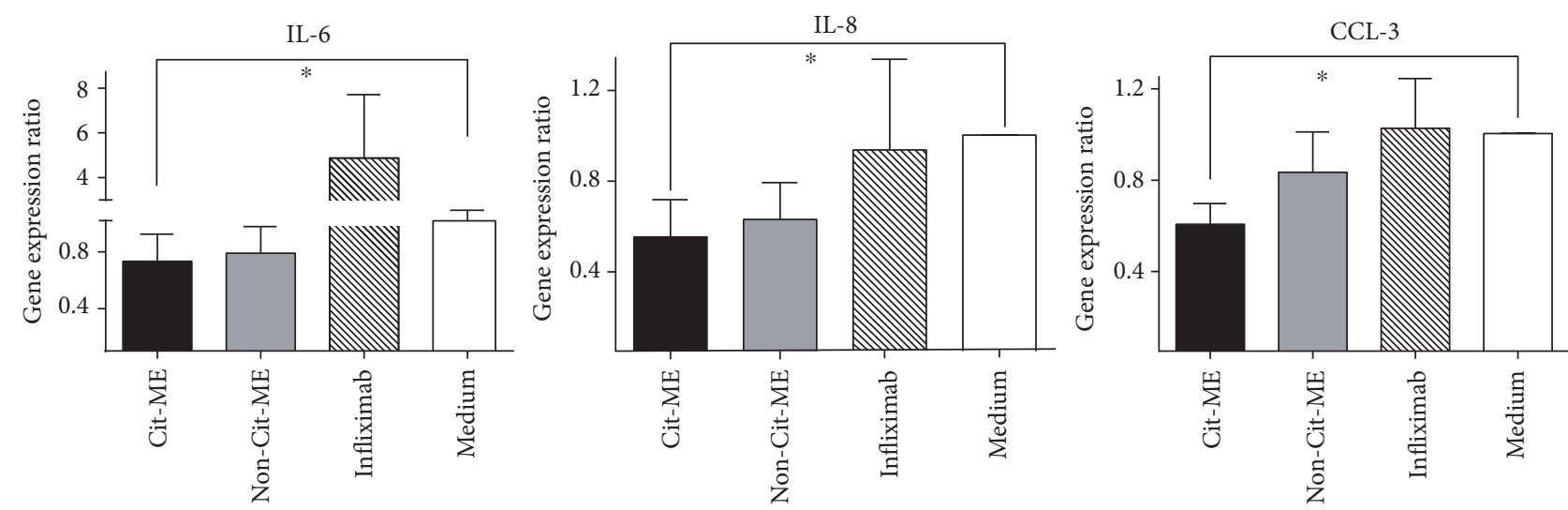

FIGURE 3: Cit-ME peptide downregulates IL-6, IL-8, and CCL-3 mRNA expression. PBMC of RA patients $(n=7-10)$ were cultured $\left(5 \times 10^{5}\right.$ cells/well) for 24 hours in the presence of Cit-ME $(1.25 \mu \mathrm{g} / \mathrm{mL})$. mRNA gene expression was determined by real-time RT-PCR. Results are presented as the mean \pm SE percentage of gene expression, ${ }^{*} p<0.02$.

medium alone, but as compared to $\beta$-Fib-NC $(n=6$, average of $1.1 \pm 0.04)$, variation was nonsignificant. Neither infliximab $(n=10$, average of $1.07 \pm 0.05)$ nor tocilizumab $(n=11$, average of $0.99 \pm 0.05)$ significantly altered the fold change of $\% \mathrm{CD}^{+} \mathrm{Foxp}^{+} \mathrm{T}$ cells compared to the medium control. Higher expression of Foxp3 on gated CD4 T cells in PBMC of representative RA patient that were incubated with Cit-ME compared to medium alone is shown (Figure 4(c)). This was true also for $\mathrm{CD}^{+} \mathrm{CD} 25^{+} \mathrm{Foxp}^{+}$ cells that were elevated in RA patients' PBMC incubated with Cit-ME (8.3\%) compared to Non-Cit-ME or medium control (7.4\%) (Figure 4(d)).

Figure 5(a) shows representative plot of $\mathrm{ACPA}^{+}-\mathrm{RA}$ patient PBMC stained with anti-CD3 and anti-IL-17. As shown in Figure 5(b), after incubation with the Cit-ME peptide, significant reduction in the fold change of $\%$ $\mathrm{CD}^{+}{ }^{+} \mathrm{IL}-17^{+} \mathrm{T}$ cells was detected $(n=9$, average of 0.77 $\pm 0.08)$ compared to the medium control $(n=9$, considered as 1) $(p=0.05)$. Incubation with the Non-Cit-ME peptide resulted in reduction in the fold change of $\% \mathrm{CD}^{+} \mathrm{IL}-17^{+} \mathrm{T}$ cells $(n=7$, average of $0.9 \pm 0.1)$ as compared to the medium control alone, but the variations were nonsignificant.

\section{Discussion}

We have shown that Cit-ME, a multiepitope citrullinated peptide, has the capacity to immunomodulate pro- and anti-inflammatory gene expression in RA patients' PBMC. In vitro Cit-ME induced Treg and decreased the number of Th17 cells. In a previous study, we demonstrated that the tolerogenic Cit-ME peptide ameliorated manifestations of arthritis in a rat model of adjuvant-induced arthritis (AIA). The mechanisms leading to suppression of arthritis by Cit-ME peptide in vivo were also mediated by altering the Treg/Th17 balance and by increasing $\mathrm{T}$ cell apoptosis [17].

ACPA are known to appear years prior to arthritis, and citrullinated proteins are assumed to be the original target antigens in RA and perhaps the immunogens [22]. Hence, they might be the key to restoring immune tolerance and prevent impending disease. The approach we present here is based on inducing peripheral tolerance using a synthetic peptide derived from sequences of multiple prevalent citrullinated autoantigens. Indeed, the citrullinated $\beta$-fibrinogen peptide was also able to immunomodulate immune cells of RA patients even if to a limited extent compared to the Cit-ME peptide.

Stimulation of PBMC from 51 RA patients with the 5 different citrullinated fibrinogen peptides $(50 \mu \mathrm{g} / \mathrm{mL})$ demonstrated that they significantly suppressed the $\mathrm{T}$ cell proliferative responses and did not change the production levels of IFN- $\gamma$ and IL-17 [23]. These findings clearly indicated that the citrullinated fibrinogen peptides did not induce an immunogenic response but rather generated a tolerogenic phenotype.

Moreover, in a clinical trial conducted on $\mathrm{ACPA}^{+}-\mathrm{RA}$ patients carrying HLA-DRB1 SE allele's, immunotherapy of autologous dendritic cells loaded with four different citrullinated peptide antigens improved a patient's clinical state. The observed effects were associated with reduction of effector $\mathrm{T}$ cells and reduced proinflammatory cytokines in the serum with an increased ratio of regulatory to effector T cells [24].

On the other hand, studies that evaluated immune responses towards different citrullinated proteins, such as citrullinated vimentin and citrullinated aggrecan in RA patients, demonstrated a certain degree of immune cell stimulation directed to these citrullinated proteins $[25,26]$.

The dose of autoantigen used is a critical parameter, vimentin and aggrecan citrullinated peptides could induce certain proliferative response in T cells from RA patients at a concentation range of $2.5-10 \mu \mathrm{g} / \mathrm{mL}$ [20]. Also, $5 \mu \mathrm{g} / \mathrm{mL}$ of TSH peptides induce $\mathrm{T}$ cell response in vitro in PBMC derived from Graves' disease patients [21]. Here, citrullinated peptides were administered at a concentration of $1.25 \mu \mathrm{g} / \mathrm{mL}$ chosen on a basis of their potential ability of tolerance induction. Possibly, such an approach induces TCR signals that are insufficient to fully activate $\mathrm{T}$ cells and, therefore, lead to functional $\mathrm{T}$ cell suppression by either energy or clonal deletion [27].

It was shown that RA patients have significantly higher frequencies of Cit-specific T cells and that a greater proportion of these cells displays a Th1 memory phenotype [28]. 


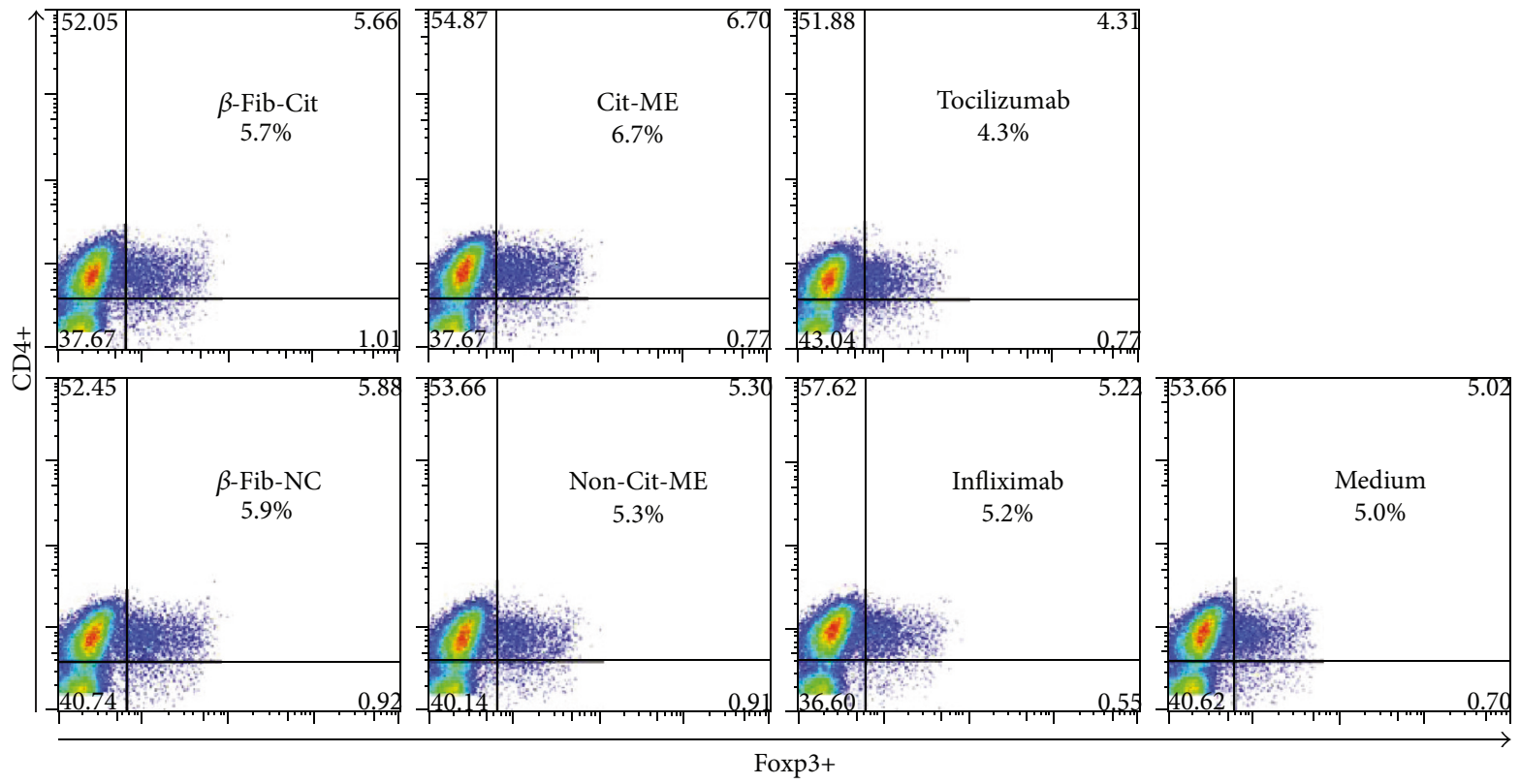

(a)

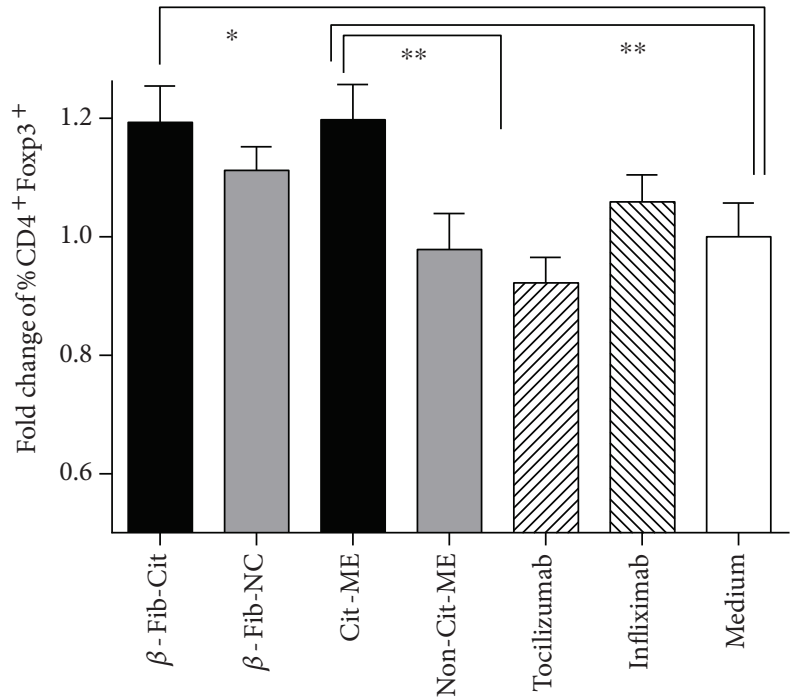

(b)
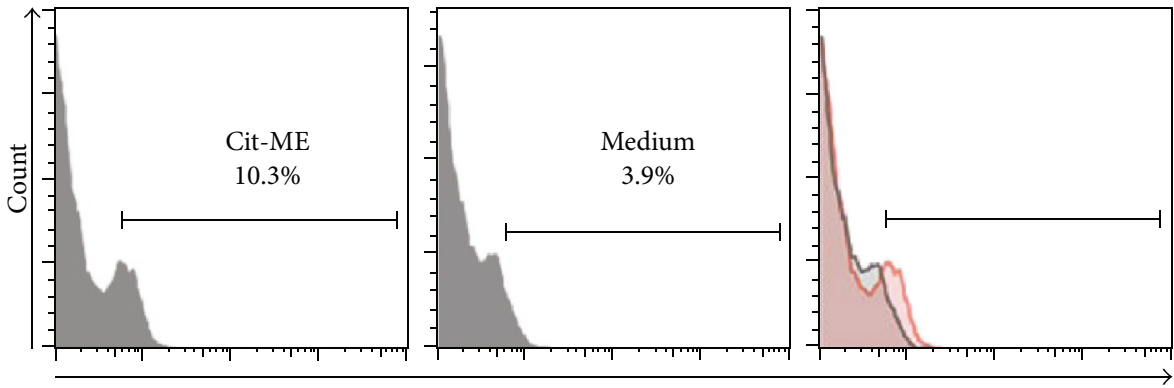

Foxp3+

Cit-ME

Medium

(c)

Figure 4: Continued. 


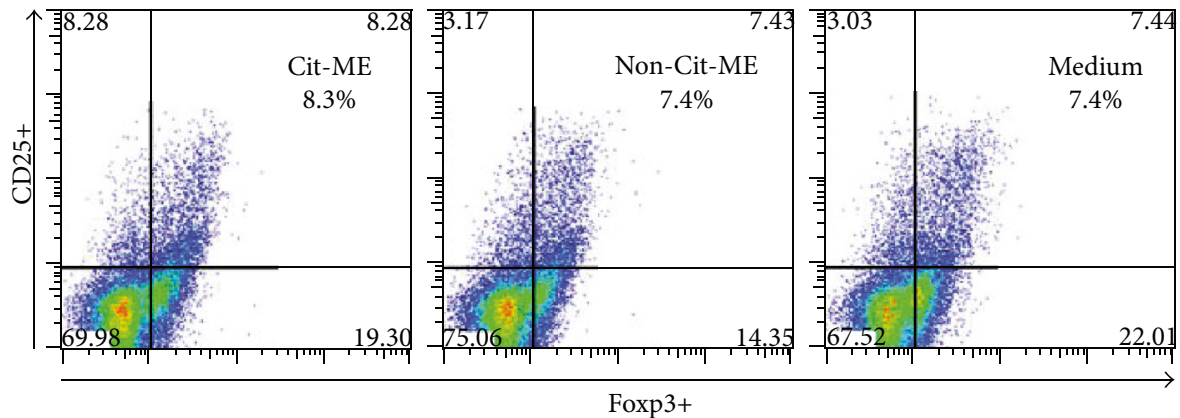

(d)

Figure 4: Cit-ME upregulates Treg in vitro. (a) Representative dot plot of $\mathrm{CD}^{+} \mathrm{Foxp}^{+} \mathrm{T}$ cells from a RA patient. Positive staining is presented in the right upper quadrant of each graph with the percentage indicated. (b) The fold change of $\%$ CD $4^{+}$Foxp $3^{+} \mathrm{T}$ cells determined by flow cytometry $(n=6-13)$. Data are presented as mean values $\left({ }^{*} p=0.04,{ }^{* *} p=0.02\right)$. (c) Histogram shows representative Foxp3 staining for one RA patient, gated for $\mathrm{CD}^{+} \mathrm{T}$ cells. (d) Representative dot plot of RA patient PBMC gated for CD4 and analyzed for CD25 and Foxp3 expression.

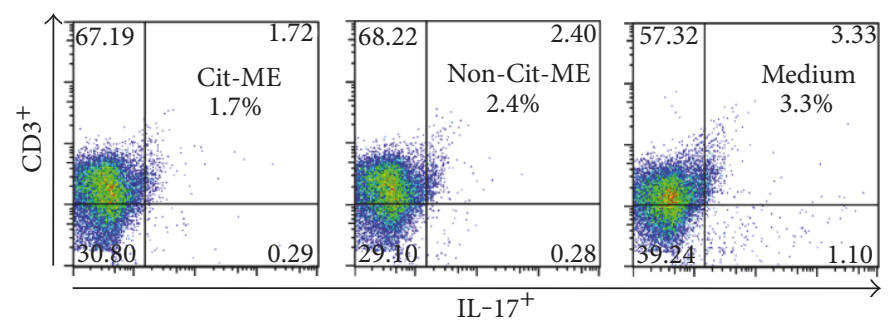

(a)

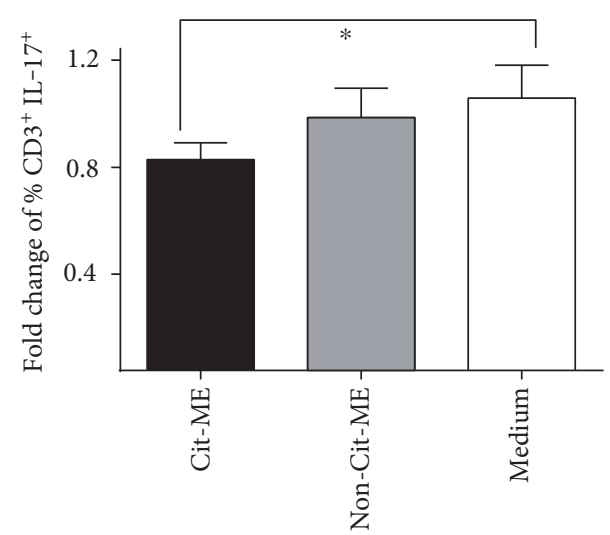

(b)

FIgure 5: Cit-ME downregulated Th17 cells in vitro. (a) Representative plots of CD3 and IL-17 staining. Positive staining is presented in the right upper quadrant of each plot with the percentage indicated. (b) Fold change of $\%$ CD3 ${ }^{+} \mathrm{IL}_{-}-17^{+} \mathrm{T}$ cells $(n=11)$. Data are presented as mean values $\left({ }^{*} p=0.05\right)$.

Thus, the administration of low-dose citrullinated peptides might assist to reverse the pathogenic autoreactive immune response by inducing immunomodulation.

We previously reported that Cit-ME effectively inhibited the course of AIA in Lewis rats. These therapeutic effects were associated with increased Treg and $\mathrm{T}$ cell apoptosis and fewer Th17 cells in the treated rats [17].

Here, the in vitro effect of Cit-ME on $\mathrm{CD}^{+}{ }^{+} \mathrm{Foxp}^{+}$and $\mathrm{CD}^{+} \mathrm{IL}-17^{+} \mathrm{T}$ cells was assessed; Cit-ME and $\beta$-Fib-Cit upregulated the $\mathrm{CD} 4^{+} \mathrm{Foxp}^{+} \mathrm{T}$ cells. Further, characterizing the suppressive capability of such population is required. Phenotypical analysis of Treg into subgroups of activated Treg (CD45RA ${ }^{-}$Foxp ${ }^{\text {high }}$ ), resting Treg $\left(\mathrm{CD}^{2} 5 \mathrm{RA}^{+}\right.$Foxp3low), which are both suppressive in vitro, and cytokinesecreting nonsuppressive non-Treg $\left(\mathrm{CD} 45 \mathrm{RA}^{-} \mathrm{FoxP} 3^{\text {low }}\right)$ as shown could be more informative [29].

For instance, heat shock protein 60 , altered peptide ligand (APL1), an additional autoantigen possibly involved in the pathogenesis of RA, was used for the induction of peripheral tolerance. Stimulation of PBMC from RA patients by APL1 increased the proportions of Treg [30], induced apoptosis of activated $\mathrm{CD}^{+} \mathrm{T}$ cells, presumably through a Tregdependent mechanism [31], and increased Treg suppression against APL1 responding effector T cells, at the same time decreased IL-17 that is produced by activated T cells [32].

Similarly, in systemic lupus erythematosus (SLE), abnormal apoptosis may lead to extracellular release of nucleosomes, which include various histone proteins that are recognized as neoepitopes [33, 34]. Supplementing PBMC cultures of lupus patients with histone nucleosomal peptides induced an increase of Treg and suppressed IFN$\alpha$ gene expression. Each of the histone peptidic epitopes also inhibited the pathogenic $\operatorname{IgG}$ autoantibody production in the lupus patients' PBMC. Combinations of these epitopes were even more efficient in suppressing pathogenic autoantibody production [35]. Accordingly, administration of low-dose histone-nucleosomal peptides induced tolerance in humans [36].

In our study, the anti-inflammatory response phenomenon exerted by the citrullinated peptides was compared to 
approved biological medications in RA (infliximab and tocilizumab). Addition of the anti-TNF- $\alpha$ agent infliximab did not affect significantly TGF- $\beta$, TNF- $\alpha$, IL- 8 , and CCL-3 gene expression, while IL- $1 \beta$ mRNA was significantly decreased and IL-6 mRNA was elevated compared to the medium control. The observed effects on lymphocytes following stimulation with infliximab can indicate on the potential drug induce target genes in naïve RA patients' PBMC. We could not detect changes in Treg population following the addition of an anti-TNF- $\alpha$ (infliximab) or an anti-IL6 receptor agent (tocilizumab). However, anti-TNF therapy in RA patients was shown to have the capacity to generate newly differentiated population of Treg cells [37].

Study limitations are the following: first, the immunomodulatory effects of the citrullinated peptides were compared to the noncitrullinated matched peptides. Although the latter peptides could not promote equivalent immunomodulation, we cannot exclude that such control peptides may have a biological effect since they possess sequence homologies to known autoantigens and might evoke certain immune responses. Scrambled peptides should be considered as a better control for Cit-ME and $\beta 60-74-F i b-C i t$ activity.

Second, the citrullinated peptides administered in our study could immunomodulate cytokine expression also in ACPA seronegative patients $(n=3)$, indicating that although the ACPA titers measured were below the cut-off of the commercial ELISA kit for ACPA detection, the citrullinated peptides could nevertheless stimulate the PBMC and alter the mRNA profile of the cytokines. Further studies are required to determine the mechanistic effects of citrullinated peptides in ACPA seronegative RA patients.

\section{Conclusion}

In the present study, we have shown that a multiepitope citrullinated peptide could be an attractive target for therapeutic intervention in RA patients. This peptide may stimulate the expression of anti-inflammatory cytokines and downregulate proinflammatory cytokines in $\mathrm{T}$ cells. These modifications may shift the Treg/Th17 imbalance and restore homeostasis in arthritis.

\section{Conflicts of Interest}

The authors have no financial conflicts of interest.

\section{Acknowledgments}

The authors thank the patients who participated in this study, and they want to acknowledge Dr. Boris Gilburd for preforming the ELISA using QUANTA Lite CCP3 (INOVA Diagnostics Inc.).

\section{References}

[1] J. B. Imboden, "The immunopathogenesis of rheumatoid arthritis," Annual Review of Pathology, vol. 4, pp. 417-434, 2009.

[2] M. M. Nielen, D. van Schaardenburg, H. W. Reesink et al., "Specific autoantibodies precede the symptoms of rheumatoid arthritis: a study of serial measurements in blood donors," Arthritis and Rheumatism, vol. 50, no. 2, pp. 380-386, 2004.

[3] P. F. Whiting, N. Smidt, J. A. Sterne et al., "Systematic review: accuracy of anti-citrullinated peptide antibodies for diagnosing rheumatoid arthritis," Annals of Internal Medicine, vol. 152, no. 7, pp. 456-464, 2010, W155-466.

[4] A. Willemze, S. Bohringer, R. Knevel et al., "The ACPA recognition profile and subgrouping of ACPA-positive RA patients," Annals of the Rheumatic Diseases, vol. 71, no. 2, pp. 268-274, 2012.

[5] C. Masson-Bessiere, M. Sebbag, E. Girbal-Neuhauser et al., "The major synovial targets of the rheumatoid arthritisspecific antifilaggrin autoantibodies are deiminated forms of the alpha- and beta-chains of fibrin," Journal of Immunology, vol. 166, no. 6, pp. 4177-4184, 2001.

[6] M. Simon, E. Girbal, M. Sebbag et al., "The cytokeratin filament-aggregating protein filaggrin is the target of the so-called "antikeratin antibodies," autoantibodies specific for rheumatoid arthritis," The Journal of Clinical Investigation, vol. 92, no. 3, pp. 1387-1393, 1993.

[7] E. R. Vossenaar, N. Despres, E. Lapointe et al., "Rheumatoid arthritis specific anti-Sa antibodies target citrullinated vimentin," Arthritis Research \& Therapy, vol. 6, no. 2, pp. R142R150, 2004.

[8] R. Engelmann, A. Biemelt, A. Cordshagen, A. Johl, D. Kuthning, and B. Muller-Hilke, "The prerequisites for central tolerance induction against citrullinated proteins in the mouse," PloS One, vol. 11, no. 6, article e0158773, 2016.

[9] I. Auger, M. Sebbag, C. Vincent et al., "Influence of HLA-DR genes on the production of rheumatoid arthritis-specific autoantibodies to citrullinated fibrinogen," Arthritis and Rheumatism, vol. 52, no. 11, pp. 3424-3432, 2005.

[10] M. A. Gonzalez-Gay, C. Garcia-Porrua, and A. H. Hajeer, "Influence of human leukocyte antigen-DRB1 on the susceptibility and severity of rheumatoid arthritis," Seminars in Arthritis and Rheumatism, vol. 31, no. 6, pp. 355-360, 2002.

[11] P. K. Gregersen, J. Silver, and R. J. Winchester, "The shared epitope hypothesis. An approach to understanding the molecular genetics of susceptibility to rheumatoid arthritis," Arthritis and Rheumatism, vol. 30, no. 11, pp. 1205-1213, 1987.

[12] J. A. Hill, S. Southwood, A. Sette, A. M. Jevnikar, D. A. Bell, and E. Cairns, "Cutting edge: the conversion of arginine to citrulline allows for a high-affinity peptide interaction with the rheumatoid arthritis-associated HLA-DRB1 ${ }^{*} 0401$ MHC class II molecule," Journal of Immunology, vol. 171, no. 2, pp. 538-541, 2003.

[13] S. C. Law, S. Street, C. H. Yu et al., "T-cell autoreactivity to citrullinated autoantigenic peptides in rheumatoid arthritis patients carrying HLA-DRB1 shared epitope alleles," Arthritis Research \& Therapy, vol. 14, no. 3, p. R118, 2012.

[14] A. H. van der Helm-van Mil, K. N. Verpoort, F. C. Breedveld, T. W. Huizinga, R. E. Toes, and R. R. de Vries, "The HLA-DRB1 shared epitope alleles are primarily a risk factor for anti-cyclic citrullinated peptide antibodies and are not an independent risk factor for development of rheumatoid arthritis," Arthritis and Rheumatism, vol. 54, no. 4, pp. 1117 1121, 2006.

[15] C. C. Striebich, M. T. Falta, Y. Wang, J. Bill, and B. L. Kotzin, "Selective accumulation of related CD4+ T cell clones in the synovial fluid of patients with rheumatoid arthritis," Journal of Immunology, vol. 161, no. 8, pp. 4428-4436, 1998. 
[16] S. Turunen, M. K. Koivula, J. Melkko, E. Alasaarela, P. Lehenkari, and J. Risteli, "Different amounts of proteinbound citrulline and homocitrulline in foot joint tissues of a patient with anti-citrullinated protein antibody positive erosive rheumatoid arthritis," Journal of Translational Medicine, vol. 11, p. 224, 2013.

[17] S. Gertel, G. Serre, Y. Shoenfeld, and H. Amital, "Immune tolerance induction with multiepitope peptide derived from citrullinated autoantigens attenuates arthritis manifestations in adjuvant arthritis rats," Journal of Immunology, vol. 194, no. 12, pp. 5674-5680, 2015.

[18] S. Gertel, Y. Shoenfeld, and H. Amital, "Tolerogenic citrullinated peptide for arthritis," Oncotarget, vol. 6, no. 23, pp. 19344-19345, 2015.

[19] C. Iobagiu, A. Magyar, L. Nogueira et al., "The antigen specificity of the rheumatoid arthritis-associated ACPA directed to citrullinated fibrin is very closely restricted," Journal of Autoimmunity, vol. 37, no. 4, pp. 263-272, 2011.

[20] A. Aggarwal, R. Srivastava, and S. Agrawal, "T cell responses to citrullinated self-peptides in patients with rheumatoid arthritis," Rheumatology International, vol. 33, no. 9, pp. 2359-2363, 2013.

[21] N. Tandon, M. A. Freeman, and A. P. Weetman, "T cell responses to synthetic TSH receptor peptides in Graves' disease," Clinical and Experimental Immunology, vol. 89, no. 3, pp. 468-473, 1992.

[22] M. Sebbag, S. Chapuy-Regaud, I. Auger et al., "Clinical and pathophysiological significance of the autoimmune response to citrullinated proteins in rheumatoid arthritis," Joint, Bone, Spine, vol. 71, no. 6, pp. 493-502, 2004.

[23] K. Shin, S. Hong, E. H. Choi et al., "Role of citrullinated fibrinogen peptides in the activation of CD4 T cells from patients with rheumatoid arthritis," Immune Network, vol. 13, no. 4, pp. 116-122, 2013.

[24] H. Benham, H. J. Nel, S. C. Law et al., "Citrullinated peptide dendritic cell immunotherapy in HLA risk genotype-positive rheumatoid arthritis patients," Science Translational Medicine, vol. 7, no. 290, p. 290ra287, 2015.

[25] A. L. Feitsma, E. I. van der Voort, K. L. Franken et al., "Identification of citrullinated vimentin peptides as T cell epitopes in HLA-DR4-positive patients with rheumatoid arthritis," Arthritis and Rheumatism, vol. 62, no. 1, pp. 117-125, 2010.

[26] A. von Delwig, J. Locke, J. H. Robinson, and W. F. Ng, "Response of Th17 cells to a citrullinated arthritogenic aggrecan peptide in patients with rheumatoid arthritis," Arthritis and Rheumatism, vol. 62, no. 1, pp. 143-149, 2010.

[27] D. M. Turley and S. D. Miller, "Prospects for antigen-specific tolerance based therapies for the treatment of multiple sclerosis," Results and Problems in Cell Differentiation, vol. 51, pp. 217-235, 2010.

[28] E. A. James, M. Rieck, J. Pieper et al., "Citrulline-specific Th1 cells are increased in rheumatoid arthritis and their frequency is influenced by disease duration and therapy," Arthritis \& Rhematology, vol. 66, no. 7, pp. 1712-1722, 2014.

[29] M. Miyara, Y. Yoshioka, A. Kitoh et al., "Functional delineation and differentiation dynamics of human CD4+ $\mathrm{T}$ cells expressing the FoxP3 transcription factor," Immunity, vol. 30, no. 6, pp. 899-911, 2009.

[30] C. Dominguez Mdel, N. Lorenzo, A. Barbera et al., “An altered peptide ligand corresponding to a novel epitope from heatshock protein 60 induces regulatory $\mathrm{T}$ cells and suppresses pathogenic response in an animal model of adjuvant-induced arthritis," Autoimmunity, vol. 44, no. 6, pp. 471-482, 2011.

[31] A. Barbera, N. Lorenzo, G. Garrido et al., "APL-1, an altered peptide ligand derived from human heat-shock protein 60 , selectively induces apoptosis in activated CD4+ CD25 $+\mathrm{T}$ cells from peripheral blood of rheumatoid arthritis patients," International Immunopharmacology, vol. 17, no. 4, pp. 1075-1083, 2013.

[32] A. Barbera, N. Lorenzo, P. van Kooten et al., "APL1, an altered peptide ligand derived from human heat-shock protein 60, increases the frequency of Tregs and its suppressive capacity against antigen responding effector CD $4+\mathrm{T}$ cells from rheumatoid arthritis patients," Cell Stress \& Chaperones, vol. 21, no. 4, pp. 735-744, 2016.

[33] H. Chabre, Z. Amoura, J. C. Piette, P. Godeau, J. F. Bach, and S. Koutouzov, "Presence of nucleosome-restricted antibodies in patients with systemic lupus erythematosus," Arthritis and Rheumatism, vol. 38, no. 10, pp. 1485-1491, 1995.

[34] L. Lu, A. Kaliyaperumal, D. T. Boumpas, and S. K. Datta, "Major peptide autoepitopes for nucleosome-specific T cells of human lupus," The Journal of Clinical Investigation, vol. 104, no. 3, pp. 345-355, 1999.

[35] L. Zhang, A. M. Bertucci, R. Ramsey-Goldman, E. R. HarshaStrong, R. K. Burt, and S. K. Datta, "Major pathogenic steps in human lupus can be effectively suppressed by nucleosomal histone peptide epitope-induced regulatory immunity," Clinical Immunology, vol. 149, no. 3, pp. 365-378, 2013.

[36] H. K. Kang, M. A. Michaels, B. R. Berner, and S. K. Datta, "Very low-dose tolerance with nucleosomal peptides controls lupus and induces potent regulatory T cell subsets," Journal of Immunology, vol. 174, no. 6, pp. 3247-3255, 2005.

[37] S. Nadkarni, C. Mauri, and M. R. Ehrenstein, "Anti-TNFalpha therapy induces a distinct regulatory $\mathrm{T}$ cell population in patients with rheumatoid arthritis via TGF-beta," The Journal of Experimental Medicine, vol. 204, no. 1, pp. 33-39, 2007. 


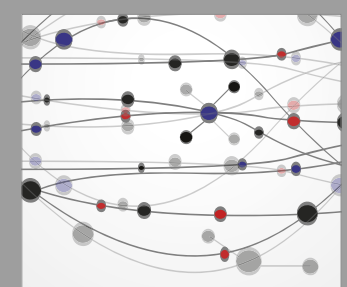

The Scientific World Journal
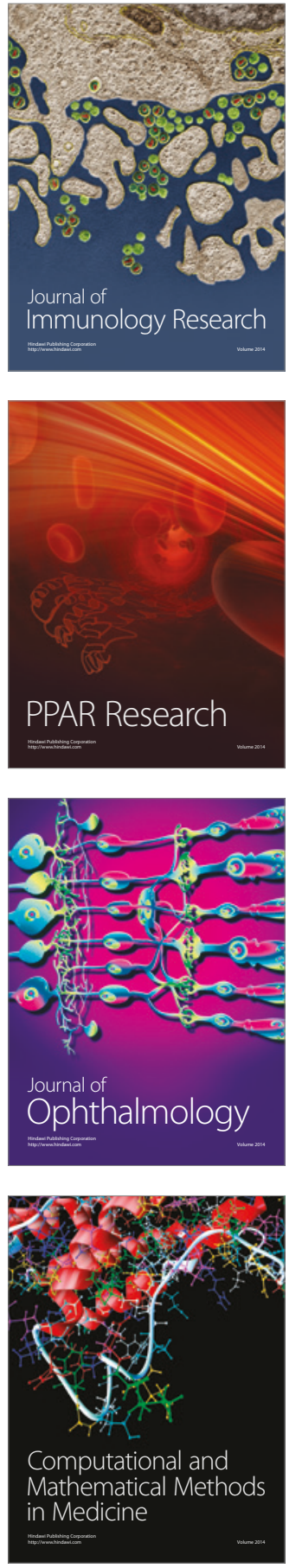

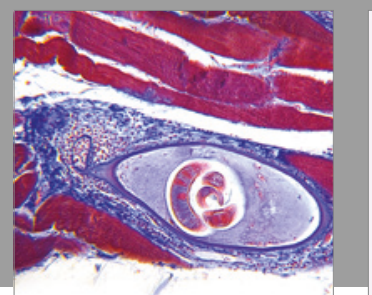

Gastroenterology Research and Practice
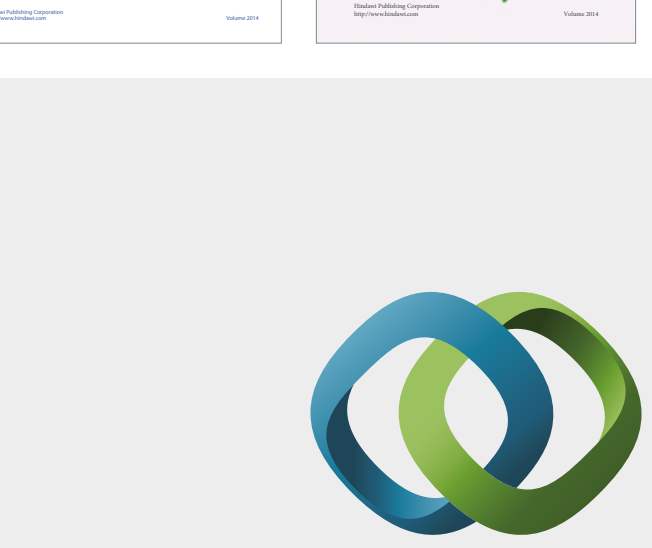

\section{Hindawi}

Submit your manuscripts at

https://www.hindawi.com
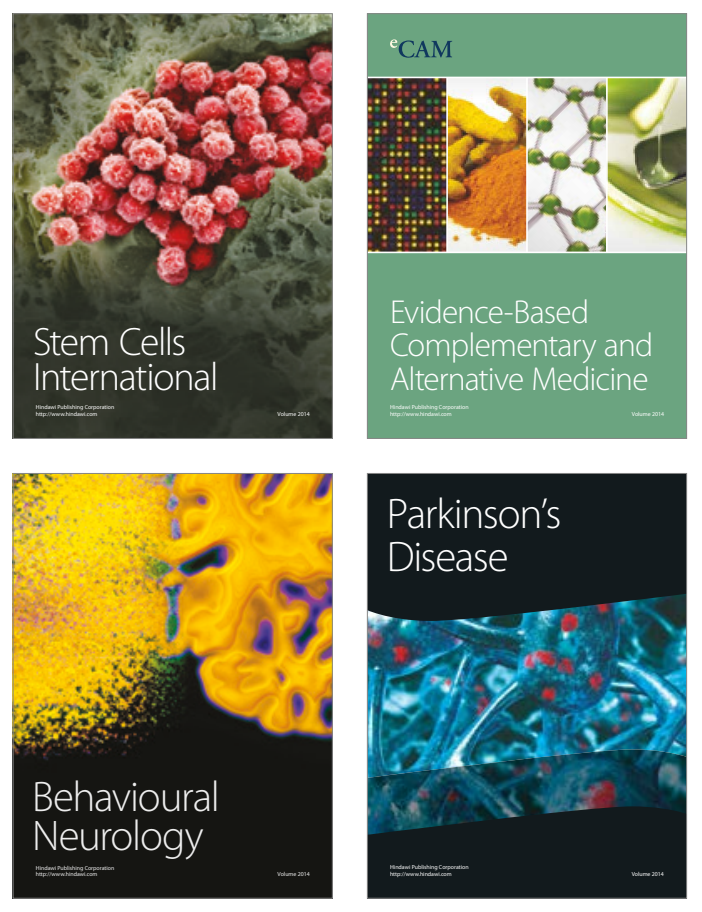
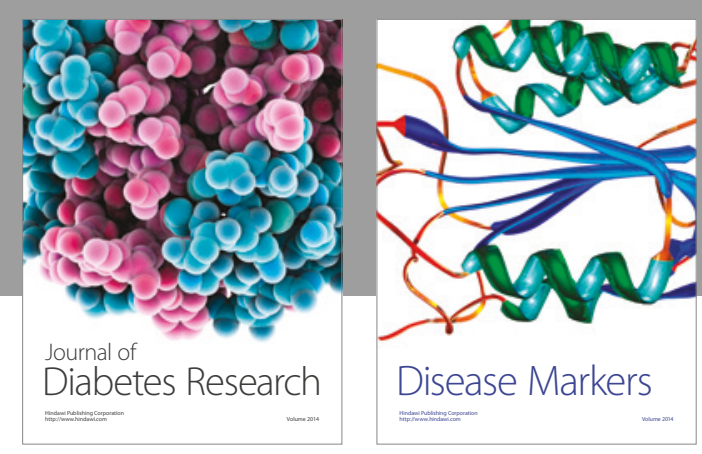

Disease Markers
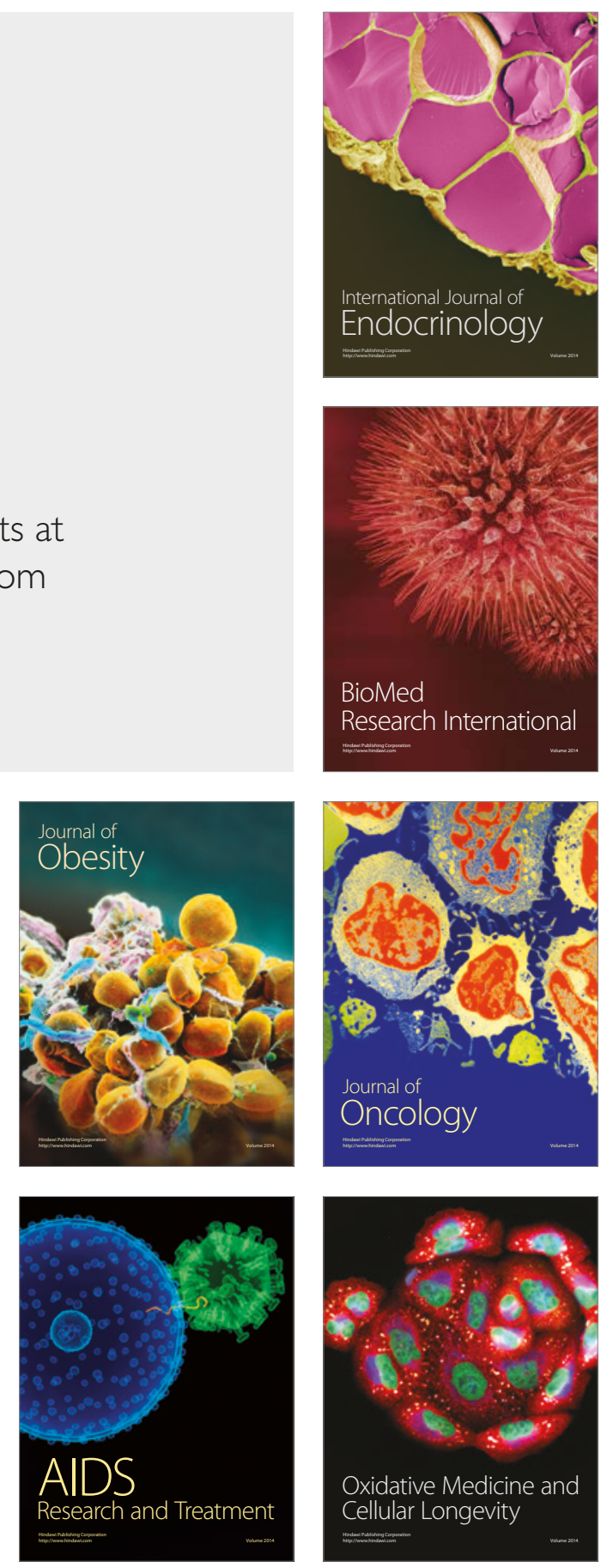